\title{
Liquid-Based Endometrial Cytology in the Management of Sonographically Thickened Endometrium
}

\author{
Anna M. Buccoliero, M.D. ${ }_{i}{ }^{1 *}$ Chiara F. Gheri, M.D., ${ }^{1}$ Francesca Castiglione, M.D., ${ }^{1}$ \\ Francesca Garbini, M.D., ${ }^{1}$ Massimiliano Fambrini, M.D., ${ }^{2}$ Gianni Bargelli, M.D., ${ }^{2}$ \\ Alfredo Barbetti, ${ }_{5}^{1}$ Salvatore Pappalardo, M.D., ${ }^{3}$ Antonio Taddei, M.D., \\ Vieri Boddi, M.D.,' Gian F. Scarselli, M.D., 2 Mauro Marchionni, M.D., ${ }^{2}$ and \\ Gian L. Taddei, M.D. ${ }^{1}$
}

Liquid-based cytology represents an opportunity to re-evaluate endometrial cytology. We evaluated the accuracy of liquid-based endometrial cytology as compared to biopsy in 670 women scheduled for histeroscopy because of thickened endometrium $(>4 \mathrm{~mm})$, as evaluated by transvaginal sonography. Endometrial biopsy detected pathology in 41 (6\%) of cases $(21$ of which were adenocarcinomas). Cytologic study found pathology in 62 (9\%) cases (19 of which were adenocarcinomas). Two hundred ninety-one biopsies (43\%) and $28(4 \%)$ cytologies were inadequate. The sensitivity and the specificity were estimated, respectively, at $95 \%$ and $98 \%$; the positive and negative predictive values were estimated, respectively, at $83 \%$ and $99 \%$. Cytology provided sufficient material more often than biopsy $(P<0.01)$. We consider endometrial cytology an efficacious diagnostic opportunity. It could be usefully applied in association with transvaginal sonography. The combination of these procedures might reduce more invasive and expensive diagnostic procedures. Diagn. Cytopathol. 2007;35:398-402. @ 2007 Wiley-Liss, Inc.

Key Words: thin-layer; liquid-based; endometrial cytology; endometrial adenocarcinoma; endometrial hyperplasia

Endometrial adenocarcinoma is the most common malignancy of the female genital tract. A significant increase in

\footnotetext{
${ }^{1}$ Department of Human Pathology and Oncology, University of Florence, Florence, Italy

${ }^{2}$ Department of Gynecology, Perinatology and Human Reproduction, University of Florence, Florence, Italy

${ }^{3}$ Private Gynecologist, Azienda Sanitaria Firenze, Florence, Italy

${ }^{4}$ Department of General Surgery, University of Florence, Florence, Italy

${ }^{5}$ Department of Statistics, University of Florence, Florence, Italy

*Correspondence to: Anna Maria Buccoliero, Department of Human Pathology and Oncology, University of Florence, Viale G.B. Morgagni, 85, 50134 Florence, Italy. E-mail: ambuccoliero@unifi.it

Received 1 May 2006; Accepted 4 May 2007

DOI 10.1002/dc.20655

Published online in Wiley InterScience (www.interscience.wiley.com).
}

its frequency, both absolute and relative, has been observed during the last decades. This increase is considered the consequence of the extended life expectancy of women, the increase in the incidence of risk factors, and of the reduction of invasive cervical carcinomas. Nowadays, more than 1 in 20 female cancers in Europe are of the endometrium. ${ }^{1-10}$

Contrary to cervical carcinoma, no screening programs have been organized for the early detection of endometrial adenocarcinoma. The lack of screening programs may be related to the precocious presenting symptoms and to the relative good prognosis of the majority of endometrial adenocarcinomas. Early abnormal uterine bleeding, the commonest presenting symptom for the $95 \%$ of women, allows diagnosis at stage I in $73 \%$ of cases. ${ }^{11}$ Moreover, there has not been an endometrial test comparable to cervicovaginal cytology for diagnostic reliability, tolerability, and low cost.

Several diagnostic procedures are used for investigating the endometrium, but there is no common agreement for the optimum approach in each circumstance (i.e. symptomatic vs. asymptomatic; with risk factors vs. without risk factors).

Endometrial cytology has been hampered in its dissemination by the difficulty in its interpretation due to the common presence of excess blood and overlapping cells. Liquid-based cytology represents an opportunity to reexamine endometrial cytology. We conducted the current study to correlate the results of liquid-based endometrial cytology with the results of endometrial biopsy in a group of 670 women scheduled for hysteroscopy because of thick endometrium ( $>4 \mathrm{~mm}$ ), as evaluated by transvaginal sonography (TVS). Our purpose was preliminarily to ascertain whether the association of the liquid-based endo- 


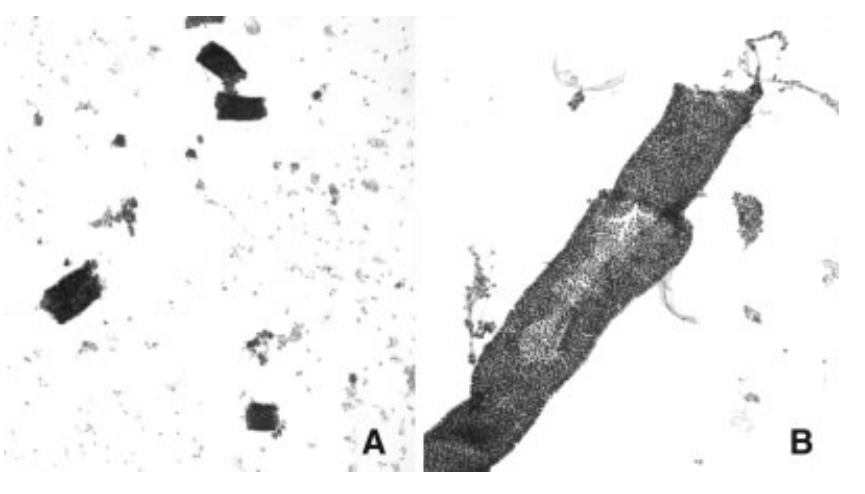

Fig. 1. Proliferative endometrium (A): three-dimensional cylindrical endometrial clusters with preserved polarity formed by isomorphic cells with scant cytoplasm and regular nuclei; secretory endometrium (B): wide three-dimensional cylindrical endometrial cluster with preserved polarity formed by isomorphic cells with wide clear cytoplasm and regular nuclei. Original magnification: $(\mathrm{A}) \times 50$; $(\mathrm{B}) \times 200$; Hematoxylin-eosin.

metrial cytology with TVS may reduce unnecessary more invasive diagnostic procedures.

\section{Study Population}

The patients included in this study were 670 women scheduled for hysteroscopy because of thickened endometrium $(>4 \mathrm{~mm})$, as evaluated by TVS in which the absence of cervical stenosis made the investigation possible. After providing informed consent, all women were submitted in sequence to hysteroscopy, endometrial cytology, and bioptic endometrial sampling.

Their median age was 53 yr (range 24-89). One hundred eight-three $(27 \%)$ women were referred for uterine bleeding and $84(13 \%)$ received hormonal therapy.

\section{Methods}

Cytological sampling was performed using the Endoflower device (RI-MOS, Mirandola, Modena, Italy; http:// www.rimos.com). After the endometrial sampling, the device tip was immersed in the Cytolyt ${ }^{\circledR}$ (Cytyc Corporation, Boxborough, MA) vial where it was vigorously rotated in order to facilitate cell release. The vial was labeled with the appropriate patient information and transported to the Department of Pathology where the samples were processed and the diagnoses were made.

The samples were centrifuged and the pellet containing the cells was transferred into a vial containing Preserv$\mathrm{Cyt}^{\circledR}$ (Cytyc Corporation). Blood and mucus were eliminated by means of washing through a succession of centrifugation and resuspension in N-Acetyl-L-Cisteine (mucolysis; before the fixation in PreservCyt) and/or acetic acid (hemolysis; after the fixation in PreservCyt). The vial was inserted into the ThinPrep 2000 automated slide processor (Cytyc Corporation). The slides were stained with routine Papanicolau (Pap) stain.

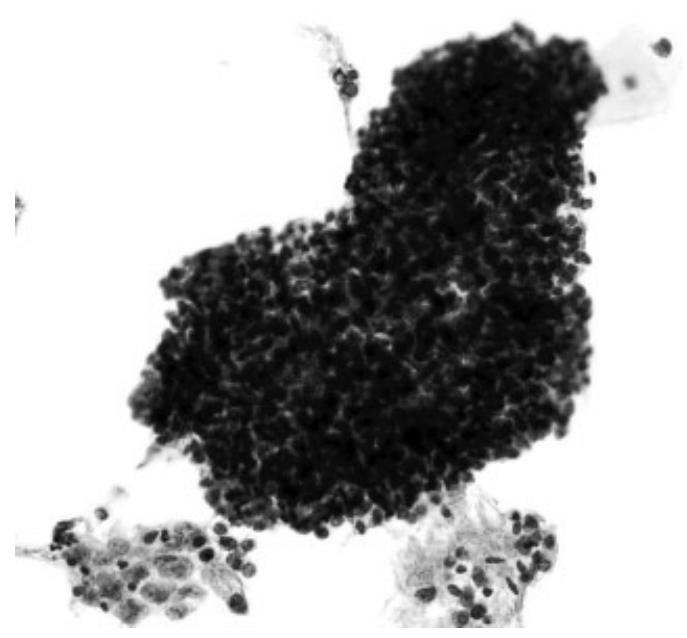

Fig. 2. Non atypical hyperplasia: wide three-dimensional clusters with considerable cellular crowding and architectural disorder. Original magnification: $\times 200$; Hematoxylin-eosin.

Histologic sampling was performed using the Endoram device (RI-MOS). Endometrial samples were routinely fixed in neutral buffered formol, embedded in paraffin, and stained with hematoxylin-eosin.

Cytological and histological diagnoses were executed blindly by two cyto-histo-gynecopathologists (AMB, GLT). In case of discordant diagnosis, both pathologists reviewed the case together and reached an agreement on the diagnosis. The slides were considered unsatisfactory when there were less than five endometrial clusters (endometrial cytology) or severe fragmentation or scarcity of the endometrial tissue (endometrial biopsy).

The cytological diagnosis was given according to the criteria that we have previously reported. ${ }^{12} \mathrm{We}$ considered four categories, normal (grouping proliferative, secretory, and atrophic endometrium), nonatypical hyperplasia, atypical hyperplasia, and carcinoma, on the basis of the architectural and cytological features of the endometrial clusters considering all anamnestic and clinical information (Figs. 1-3). The histological diagnosis was given according to the World Health Organization (WHO) criteria. $^{1}$

Because of the small number of pathologic specimens, statistical analyses were performed categorizing the cases as nonpathological and pathological (hyperplasia, atypical hyperplasia, carcinoma).

The sensitivity was calculated by dividing the true pathological cases diagnosed by endometrial cytology by the total number of patients with endometrial pathology and multiplying by 100 ; the specificity was calculated by dividing the number of true negative cases diagnosed by endometrial cytology by the total number of women who did not have endometrial disease and multiplying by 100 ; the positive predictive value was calculating by dividing 

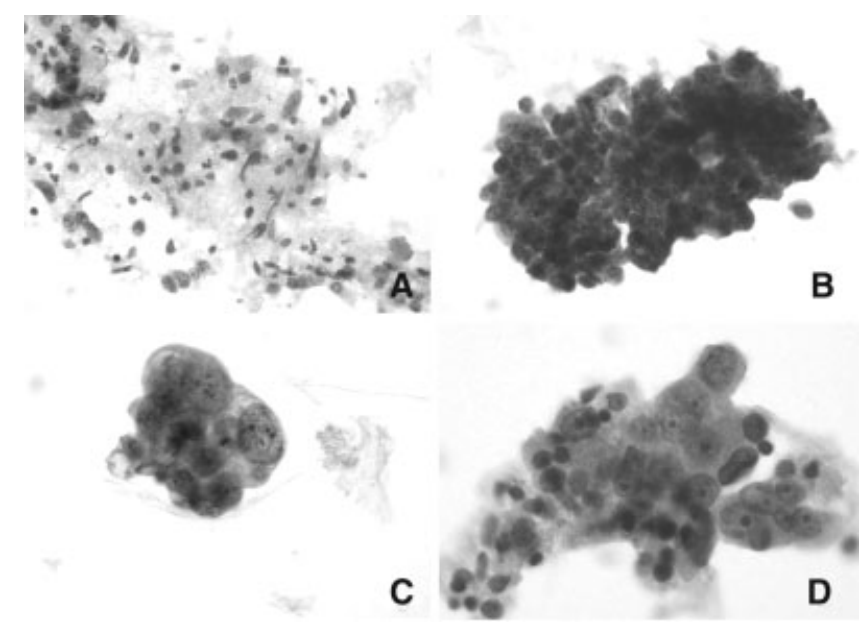

Fig. 3. Endometrial adenocarcinoma: small endometrial clusters with architectural disorder (B-D) in a phlogistic-necrotic background (A); macronucleoli and coarsely granular chromatin (D). Original magnification: (A-D) $\times 200$; Hematoxylin-eosin.

the true pathological cases by the total of true positive cytological specimens and of the false-positive cases and multiplying by 100; the negative predictive value was calculated by dividing the true negative cases by the total of true negative and of the false-negative cytological specimens and multiplying by 100 . The $\chi^{2}$ test was used to compare the number of unsatisfactory cytological specimens with that of endometrial biopsies. Statistical significance was judged as $P<0.04$. Data analysis was performed using the Glaz SA 3.03 Version statistical package (Mc Graw-Hill, 1992, New York, NY).

\section{Results}

The evaluation of the biopsies determined that $21(3 \%)$ women had adenocarcinoma, $4(<1 \%)$ adenomatous atypical hyperplasia, and $16(2 \%)$ had simple nonatypical hyperplasia. Endometrial cytology evidenced 19 (3\%) neoplastic cases, $5(<1 \%)$ hyperplastic with atypia cases, and $38(6 \%)$ hyperplastic without atypia cases (Table I).

Fifteen of $21(71 \%)$ endometrial adenocarcinomas, 2 of $4(50 \%)$ adenomatous atypical hyperplasias, and 5 of $16(31 \%)$ simple nonatypical hyperpasias, as evaluated by biopsy, occurred in women with abnormal uterine bleeding.

Two hundred ninety-one biopsies (43\%) and 28 (4\%) cytologies were inadequate. Of the 291 unsatisfactory endometrial biopsies, $263(90 \%)$ were considered inadequate by the gynecologists because of the scarcity of the sampled tissue and since no tissue was sent to the laboratory; the remaining $28(10 \%)$ inadequate cases were judged inadequate by pathologists during histopathological evaluation. In 12 cases the cytology was inadequate and the biopsy was adequate, in 275 cases the biopsy was inadequate and the cytology was inadequate, and in 16
Table I. Histological and Cytological Results (670 Women)

\begin{tabular}{lcc}
\hline & Histology, $n$ & Cytology, $n$ \\
\hline Nonpathological & $338(50)^{\mathrm{a}}$ & $580(87)$ \\
Pathological & $41(6)^{\mathrm{b}}$ & $62(9)^{\mathrm{c}}$ \\
Inadequate & $291(43)$ & $28(4)$ \\
Total & $670(100)$ & $670(100)$ \\
\hline
\end{tabular}

${ }^{\mathrm{a}}$ Values in parentheses indicate percentages.

$\mathrm{b}_{21}$ adenocarcinomas, 4 adenomatous atypical hyperplasias, 16 simple nonatypical hyperplasias.

${ }^{\mathrm{c}} 19$ adenocarcinomas, 5 atypical hyperplasias, 38 nonatypical hyperplasias.

Table II. Discordant Cases

\begin{tabular}{lll}
\hline Cases, $n$ & \multicolumn{1}{c}{ Histology } & \multicolumn{1}{c}{ Cytology } \\
\hline $8(2)^{\mathrm{a}}$ & Nonpathological & Nonatypical hyperplasia \\
$2(1)$ & Simple nonatypical hyperplasia & Nonpathological \\
$2(1)$ & Adenocarcinomas & Atypical hyperplasia \\
$2(<1)$ & Adenomatous atypical hyperplasia & Nonatypical Hyperplasia \\
\hline
\end{tabular}

${ }^{a}$ Values is parentheses indicate percentages.

cases both the cytology and the biopsy were inadequate; cytohistological correlation was possible in the remaining $367(55 \%)$ cases. In the series of 367 cases in which both endometrial biopsy and cytology were adequate, endometrial cytology recognized 19 neoplastic cases, 5 hyperplastic with atypia cases, and 23 hyperplastic without atypia cases (Table I). Two endometrial adenocarcinomas and one atypical hyperplasia were underrated by endometrial cytology, resulting respectively as atypical hyperplasias and as nonatypical hyperplasia. Two simple nonatypical hyperplasias resulted as negative by cytology. Eight cases were false-positive (nonatypical hyperplasias by cytology, negative on biopsy) (Table II). When we categorized the cases studied as nonpathological and pathological, endometrial cytology showed the sensitivity estimated at $95 \%$, the specificity at $98 \%$, the positive predictive value at $83 \%$, and the negative predictive value at $99 \%$.

Endometrial cytology provided sufficient material for the diagnosis more often than endometrial biopsy $(P<$ $0.01)$.

\section{Discussion}

Several diagnostic procedures are used to investigate the endometrium. Endometrial biopsy, particularly under hysteroscopic control, is considered the most efficacious one. However, this procedure may cause anxiety and discomfort Consequently, less invasive procedures are commonly preferred as the first diagnostic approach.

TVS is increasingly being used because it is well tolerate. Several studies showed the relationship between endometrial thickness, as measured by TVS, and endometrial pathology. However, the results on the diagnostic accuracy of TVS are conflicting also because of the difficulty to establish a reliable threshold separating normal 
from abnormally thickened endometrium. There is no unanimous agreement regarding the endometrial thickness that have to be considered pathologic. ${ }^{13-18}$ The endometrial thickness physiologically changes in relation to the age and, in cyclic women, to the endometrial phase. The administration of hormonal therapy may also influence the endometrial thickness. Thickened endometrium as evaluated by TVS may be the consequence of many pathological and nonpathological conditions different from carcinoma or hyperplasia such as endometrial polyps or cystic endometrium, which is typically induced by tamoxifen. ${ }^{13-18}$ Often, TVS does not provide a definitive diagnoses particularly due to its high false-positive rate. A recent meta-analysis of the diagnostic accuracy of endometrial thickness measurements by transvaginal or transabdominal ultrasound involving 9,031 women with postmenopausal bleeding reported that using a $\leq 5 \mathrm{~mm}$ cut-off level, a positive test raised the probability of carcinoma from $14.0 \%$ to $31.3 \%$, while a negative test reduced it to $2.5 \%{ }^{19}$ It is remarkable to note that in our series of women with thickened endometrium $(>4 \mathrm{~mm})$ we detected pathology in only 41 cases $(6 \%)$, of which 21 (3\%) were adenocarcinomas. The majority of these adenocarcinomas (15 cases) occurred in symptomatic patients. Analogously, in 2005 Tsuda et al. documented that the prevalence of endometrial disease in asymptomatic and symptomatic women whit thickened endometrium was $2.3 \%$ and $21 \% .^{17}$

Regarding traditional endometrial cytology, despite several encouraging results have been reported with sensibility and specificity reaching $96 \%,{ }^{20-24}$ its diffusion has been hampered because of technical and diagnostic difficulties.

The diagnostic capacity of endometrial cytology has improved enough because of the introduction of liquidbased method. The main characteristics of this method are the reduction of the obscuring factors, the distribution of the cells in a thin layer, and the possibility to obtain more than one slide available for further investigations, i.e. immunohistochemistry. Moreover, it has recently been suggested that cell block preparation obtained from liquid-based cytological sampling, when necessary, might further increase the diagnostic accuracy of thin-layer endometrial cytology. ${ }^{25}$

Some recent studies emphasised the diagnostic potentiality of endometrial thin-layer cytology: Garcia et al. ${ }^{26}$ in 2003 reported a very good specificity (96\%), a good (78\%) sensitivity, and a low (15\%) inadequate rate (lower than endometrial biopsy calculated at 26\%). In the same year we documented a cytohistologic concordance of $98 \%$ and a low unsatisfactory rate $(18 \%) .^{27} \ln 2005$, Papaefthimiou et al. $^{28}$ indicated that liquid-based endometrial cytology makes possible a nearly perfect interobserver and intraobserver agreement.
The low percentage of unsatisfactory specimens (4\%), the high sensitivity (95\%), specificity (98\%), and positive $(83 \%)$ and negative $(99 \%)$ predictive values found in our present study support this encouraging results.

The unusual high inadequate rate of endometrial biopsies in our collection may be related to the predominance of peri-postmenopausal women (median age was $53 \mathrm{yr}$ ) and of nonpathological cases (94\%); additionally, we speculate that in our dual sampling protocol the first sampling (in our protocol cytology) might impair the success of the second sampling (in our protocol biopsy). ${ }^{29}$

In conclusion we considered endometrial cytology an efficacious diagnostic opportunity. It could be usefully applied in association with transvaginal ultrasonography. The combination of these procedures may improve their diagnostic accuracy and reduce unnecessary more invasive and more expensive diagnostic procedures.

\section{References}

1. Tavassoli FA, Devilee P. World Health Organization classification of tumours, Tumours of the breast and female genital organs Pathology and genetics, Lyon, France. IARC Press; 2003.

2. Jick H, Walker AM, Rothman KJ. The epidemic of endometrial cancer. A commentary. Am J Public health 1980;70:264-267.

3. Dove-Edwin I, Boks D, Goff S, et al. The outcome of endometrial carcinoma surveillance by ultrasound scan in women at risk of hereditary nonpolyposis colorectal carcinoma and familial colorectal carcinoma. Cancer 2002;94:1708-1712.

4. Paley PJ. Screening for the major malignancies affecting women: Current guidelines. Am J Obstet Gynecol 2001;184: 1021-1030.

5. Kaaks R, Lukanova A, Kurzer MS. Obesity, endogenous hormones, and endometrial cancer risk: A synthetic review. Cancer Epidemiol Biomarkers Prev 2002;11:1531-1543.

6. Münstedt K, Grant P, Woenckhaus J, Roth G, Tinneberg HR. Cancer of the endometrium: Current aspects of diagnostics and treatment. World J Surg Oncol 2004;2:24 doi: 10.1186/1477-7819$2-24$.

7. Bray F, Santos Silva D, Moller H, Weiderpass E. Endometrial cancer incidence trends in Europe: Underlying determinants and prospects for prevention. Cancer Epidemiol Biomarkers Prev 2005;14: 1132-1142.

8. De Muylder X, Neven P, De Somer M, Van Belle Y, Vanderick G, De Muylder E. Endometrial lesions in patients undergoing tamoxifen therapy. Int J Gynaecol Obstet 1991;36:127-130.

9. Magriples U, Naftolin F, Schwartz PE, Carcangiu ML. High-grade endometrial carcinoma in tamoxifen-treated breast cancer patients. J Clin Oncol 1993;11:485-490.

10. Assikis VJ, Neven P, Jordan VC, Vergote I. A realistic clinical perspective of tamoxifen and endometrial carcinogenesis. Eur J Cancer 1996;32A:1464-1476.

11. Giusa-Chiferi MG, Goncalves WJ, Baracat EC, et al. Transvaginal ultrasound, uterine biopsy and hysteroscopy for postmenopausal bleeding. Int J Gynecol Obstet 1996:55:39-44.

12. Taddei GL, Buccoliero AM. Atlante di citologia endometriale. L'endometrio normale, iperplastico e neoplastico nella citologia in fase liquida. Florence, Italy: SEE Editrice; 2005.

13. Tsuda H, Kwabata M, Kawabata K, Yamamoto K, Umesaki N. Improvement of diagnostic accuracy of transvaginal ultrasound for identification of endometrial malignancies by using cutoff level of 


\section{BUCCOLIERO ET AL.}

endometrial thickness based on length of time since menopause. Gynecol Oncol 1997:64:35-37.

14. Tabor A, Watt HC, Wald NJ. Endometrial thickness as a test for endometrial cancer in women with postmenopausal vaginal bleeding. Obstet Gynecol 2002:99:663-669.

15. Gupta JK, Chien PF, Voit D, Clark TJ, Khan KS. Ultrasonographic endometrial thickness for diagnosing endometrial pathology in women with postmenopausal bleeding: A meta-analysis. Acta Obstet Gynecol Scand 2002:81:799-816.

16. Smith-Bindman R, Weiss E, Feldstein V. How thick is too thick? When endometrial thickness should prompt biopsy in postmenopausal women without vaginal bleeding? Ultrasound Obstet Gynecol 2004:24:558-565.

17. Tsuda H, Nakamura H, Inoue T, Kawamura N, Adachi K, Bandera CA. Transvaginal ultrasonography of the endometrtium in postmenopausal Japanese women. Gynecol Obstet Invest 2005;60: 218-223.

18. Weaver J, McHugo JM, Clark TJ. Accuracy of transvaginal ultrasound in diagnosing endometrial pathology in women with postmenopausal bleeding on tamoxifen. Br J Radiol 2005:78:394-397.

19. Gupta JK, Chien PF, Voit D, Clark TJ, Khan KS. Ultrasonographic endometrial thickness for diagnosing endometrial pathology in women with postmenopausal bleeding: A meta-analysis. Acta Obstet Gynecol Scand 2002:81:799-816.

20. Rascoe RR. Endometrial aspiration smear in diagnosis of malignancy of the uterine corpus. Am J Obstet Gynecol 1963;87:921-925.
21. An-Foraker SH, Kawada CY, McKinney D. Endometrial aspiration studies on Isaacs cell sampler with cytohistologic correlation. Acta Cytol 1979;23:303-308.

22. Buratti E, Cefis F, Masserini M, Goisis F, Vergadoro F, Bolis G. The value of endometrial cytology in a high risk population. Tumori 28;71:25-28.

23. Byrne AJ. Endocyte endometrial smears in the cytodiagnosis of endometrial carcinoma. Acta Cytol 1990;34:373-381.

24. LaPolla JP, Nicosia S, McCurdy C, et al. Experience with the EndoPap device for the cytologic detection of uterine cancer and its precursors: A comparision of the EndoPap with fractional curettage or hysterectomy. Am J Obstet Gynecol 1990;163:1055-1060.

25. Kyroudi A, Paefthmiou M, Symiakaki H, Mentzelopoulou P, Voulgaris Z, Zarakitsos P. Increasing diagnostic accuracy with cell block preparation from thin-layer endometrial cytology: A feasibility study. Acta Cytol 2006;50:63-69.

26. Garcia F, Barker B, Davis J, et al. Thin-layer cytology and histopathology in the evaluation of abnormal uterine bleeding. J Reprod Med 2003,48:882-888.

27. Buccoliero AM, Caldarella A, Noci I, et al. Thin layer method in endometrial cytology. Pathologica 2003;95:179-184.

28. Papaefthimiou M, Symiakaki H, Mentzelopoulou P, et al. Study on the morphology and reproducibility of the diagnosis of endometrial lesions utilizing liquid-based cytology. Cancer 2005;105:56-64.

29. Grimes DA. Diagnostic dilatation and curettage: A reappraisal. Am J Obstet Gynecol 1982;142:1-6. 